The Solar Orbiter mission Astrophysics Special issue

\title{
Editorial
}

\section{The Solar Orbiter mission}

This volume presents a series of articles on the Solar Orbiter space mission and its ten instruments. Solar Orbiter, an ESA-NASA collaboration, was launched on February 10, 2020. It carries the most comprehensive payload flown in the inner heliosphere to date, with six remote-sensing instruments that image the Sun and its surroundings as well as four in situ instruments for monitoring the immediate environment of the spacecraft. A series of Venus and Earth gravity assists will adjust the probe's perihelion to a minimum of $0.28 \mathrm{AU}$ and raise the inclination of the orbital plane to over 33 degrees. This will allow the first-ever look at the solar poles. Thus, Solar Orbiter is the conceptual combination of two missions: an out-of-ecliptic in situ probe (like Ulysses) and one that brings state-of-the-art telescopes (which are an improvement over those of, e.g., SOHO and SDO) closer to the Sun than ever before as well as over the solar poles. Solar Orbiter will address the most pressing open questions of solar physics, and its results will remain unique for at least the next decade.

All calibrated science data will be made available three months after their reception on the ground, in line with the open-data philosophy of the mission. The publication of this special issue is coordinated with the release of the first data from the four in situ instruments through the public ESA Solar Orbiter archive.

We warmly thank Yannis Zouganelis and the Solar Orbiter teams for coordinating this special feature.

Thierry Forveille, Steve Shore Astronomy $\mathcal{G}$ Astrophysics Editors 